\title{
Novos registros de Asopinae (Pentatomidae) predadores de lagartas Nystalea nyseus (Cramer, 1775) (Lepidoptera: Notodontidae)
}

\author{
Iris Cristiane Magistrali ${ }^{1 *}$ \\ Ervandil Corrêa Costa ${ }^{2}$ \\ Leonardo Mortari Machado ${ }^{2}$ \\ Janaína De Nadai ${ }^{3}$ \\ ${ }^{1}$ Universidade Federal Rural do Rio de Janeiro \\ BR 465, Km 7, Campus Universitário, CEP 23890-000, Seropédica - RJ, Brasil \\ ${ }^{2}$ Universidade Federal de Santa Maria \\ Avenida Roraima, 1000, Campus Universitário, CEP 97105-900, Santa Maria - RS, Brasil \\ ${ }^{3}$ Universidade Federal de Mato Grosso \\ Avenida Alexandre Ferronato, 1200, Setor Industrial, CEP 78550-000, Sinop - MT, Brasil \\ * Autor para correspondência \\ irismagistrali@gmail.com
}

Submetido em 09/04/2013

Aceito para publicação em 07/02/2014

\section{Resumo}

Lagartas desfolhadoras são importantes insetos pragas em plantações florestais. O objetivo deste trabalho foi registrar a ocorrência de percevejos predadores em surtos de Nystalea nyseus em plantações de eucalipto. O estudo foi realizado em uma plantação seminal de Eucalyptus saligna Smith localizada no município de São Sepé, Rio Grande do Sul. Uma inspeção visual foi realizada para coletar os potenciais predadores. As espécies predadoras de N. nyseus: Podisus nigrispinus, Brontocoris tabidus e Alcaeorrhynchus grandis todas pertencentes à ordem Heteroptera e família Pentatomidae foram registradas. Isso pode indicar a possibilidade de controle biológico aplicado com esses percevejos predadores em surtos de $N$. nyseus.

Palavras-chave: Asopinae; Inimigo natural; Lagartas desfolhadoras

\section{Abstract}

New records of Asopinae (Pentatomidae) predators of caterpillars Nystalea nyseus (Cramer, 1775) (Lepidoptera: Notodontidae). Defoliating caterpillars are important insect pests in forest plantations in Brazil. The aim of this study was to record the occurrence of predatory stinkbugs in outbreaks of Nystalea nyseus in eucalyptus plantations. The study was carried out in a seedling plantation of Eucalyptus saligna Smith in the municipality of São Sepe, Rio Grande do Sul. A visual inspection was performed to collect potential predators. The species Podisus nigrispinus, Brontocoris tabidus and Alcaeorrhynchus grandis, all belonging to the order Heteroptera and family Pentatomidae, were recorded. This may indicate the possibility of applied biological control with these predatory stinkbugs in population outbreaks of $N$. nyseus.

Key words: Asopinae; Natural enemies; Defoliating caterpillars 


\section{Introdução}

As plantações florestais representam ambientes modificados, geralmente com uma ou poucas espécies vegetais em grandes extensões de terras. Isso pode favorecer o estabelecimento de pragas, tornando necessária a utilização de diferentes técnicas de controle (GUTIERREZ, 2006). Dentre os métodos que podem ser utilizados no Manejo Integrado de Pragas, pode-se citar o controle biológico. Segundo Debach e Rosen (1991), o controle biológico consiste na regulação da densidade populacional, seja ela de plantas ou de animais por agentes de mortalidade, sejam eles patógenos, parasitoides, predadores ou competidores.

Percevejos da família Pentatomidae, sub-família Asopinae apresentam amplo potencial de regulação biótica de insetos pragas em sistemas florestais. Esse potencial é evidenciado pelo crescente interesse sobre esses organismos como agentes de controle biológico de insetos-pragas no Brasil (HOLTZ et al., 2009; TORRES; BOYD, 2009; ALMEIDA et al., 2011) o que segue uma tendência mundial (DE CLERCQ, 2000). Inúmeras espécies predadoras podem ser eficientes agentes reguladores de população, especialmente insetospragas das ordens Lepidoptera e Coleoptera, os quais consomem ovos, larvas e adultos desses insetos-pragas (RUBERSON et al., 1986; LEMOS, 2001).

No Estado do Rio Grande do Sul, têm sido realizados inúmeros trabalhos envolvendo a superfamília Pentatomoidea, dos quais, destacam-se principalmente aqueles que abrangem: estudos morfológicos (MATESCO et al., 2003; 2012), taxonômicos (BARCELLOS; GRAZIA, 2008; WEILER et al., 2011), levantamentos de percevejos e suas respectivas plantas hospedeiras (LINK; GRAZIA, 1987; COSTA et al., 1995; GRAZIA et al., 2004; GARLET et al., 2010) e diversidade de espécies (BUNDE, 2005; SCHMIDT; BARCELLOS, 2007).

Estudos em relação à Pentatomidae são ainda escassos, a despeito da ocorrência de predadores representantes dessa família que podem ser utilizados em programas de controle biológico de lagartas no Sul do Brasil. Portanto, o objetivo deste trabalho foi identificar a ocorrência de espécies de percevejos predadores de lagartas de $N$. nyseus em plantações de eucalipto.
Este estudo foi realizado em uma plantação seminal de Eucalyptus saligna Smith de dois anos e oito meses, espaçamento de 3,5 x 2,14 m em 77 ha localizados no Horto Florestal Boa Aguada, (30¹2'97’'S e 5335'63’W), município de São Sepé, Rio Grande do Sul. Os arredores da plantação apresentavam pastagem e fragmentos florestais nativo da região.

O clima da região de acordo com a classificação de Köppen é Cfa, correspondendo à Subtropical Temperado Úmido. Tem como principais características temperaturas médias anuais em torno de $17^{\circ} \mathrm{C}$, umidade relativa do ar média de $76 \%$, e o regime de chuvas ao redor de 1500 mm/ano (MORENO, 1961).

Durante os meses de abril e maio de 2011 foram realizados levantamentos para verificar a ocorrência de percevejos predadores em Eucalyptus saligna que se encontrava atacado por um surto de Nystalea nyseus, no município de São Sepé, Rio Grande do Sul.

Realizaram-se inspeções visuais nas folhas de eucalipto do terço inferior das árvores objetivando a coleta manual de todos os pentatomídeos encontrados. Os espécimes coletados foram acondicionados individualmente em potes plásticos e encaminhados para o Laboratório de Entomologia Florestal da UFSM. Durante o levantamento, foram coletados percevejos em atividade de predação sobre lagartas de N. nyseus e aqueles que se encontravam no sub-bosque.

Os percevejos coletados foram encaminhados ao Departamento de Zoologia da Universidade Federal do Rio Grande do Sul para a identificação em nível de espécie.

Visando confirmar a real condição de predador de lagartas de N. nyseus, um representante adulto de cada espécie de percevejo coletado no campo foi acondicionado em pote plástico $(20 \times 10 \mathrm{~cm})$, forrado com papel filtro, contendo um chumaço de algodão embebido em água destilada, trocadas diariamente para manter a umidade. Para avaliar a ação do predador, foram oferecidas cinco lagartas de $N$. nyseus apresentando diferentes ínstares para cada uma das espécies de predadores. Diariamente, folhas frescas de eucalipto foram oferecidas para as lagartas. Os indivíduos permaneceram em sala de criação com temperatura de $25^{\circ} \mathrm{C} \pm 1{ }^{\circ} \mathrm{C}$, umidade relativa do ar de $80 \%$ e fotoperíodo artificial de $12 \mathrm{~h}$ durante cinco dias. 
Diariamente, foram observados os potes contendo as lagartas e os percevejos, a fim de confirmar a ação predatória dos percevejos.

As observações realizadas a campo e, posteriormente, em laboratório confirmaram o ataque e consumo de lagartas de $N$. nyseus por representantes da sub-família Asopinae, (Heteroptera: Pentatomidae) das seguintes espécies predadoras: Podisus nigrispinus (Dallas, 1851) (Figura 1A), Brontocoris tabidus (Signoret, 1863) (Figura 1B) e Alcaeorrhynchus grandis (Dallas, 1851) (Figura 1C).

Torres et al. (2006) já haviam mencionado a espécie P. nigrispinus como predadora de lagartas de N. nyseus nos Estados de Minas Gerais, Espírito Santo e São Paulo. Entretanto, até o momento, não há nenhum registro no Brasil da espécie $B$. tabidus em associação com lagartas de N. nyseus, sendo este o primeiro registro.

No Brasil, a espécie P. nigrispinus tem sido criada em laboratório e liberada no campo para o controle de lepidópteros desfolhadores de diversas culturas (TORRES et al., 2006). Brontocoris tabidus, juntamente com P. nigrispinus destaca-se, no Brasil, como uma das espécies mais comuns e importantes para o controle biológico de lepidópteros desfolhadores em sistemas florestais (ZANUNCIO et al., 1994; JUSSELINO FILHO et al., 2001).

Surtos de lagartas desfolhadoras como Apatelodes sericea Schaus, 1896 (Eupterotidae), Psorocampa denticulata Schaus, 1901 (Notodontidae), Thyrinteina arnobia (Stoll, 1782) (Geometridae), Sarsina violascens
(Herrich-Schaeffer, 1856) (Lymantriidae) foram controladas com as espécies predadoras $P$. nigrispinus e B. tabidus (ZANUNCIO et al., 2002).

$\mathrm{O}$ crescente número de predadores em plantios de eucalipto pode estar associado ao surto populacional de $N$. nyseus, pois se sabe que as espécies de predadores encontradas no presente trabalho apresentam zoofitofagia, ou seja, são carnívoras e eventualmente se alimentam de plantas. Portanto, na ausência de presas, podem se alimentar da seiva de plantas ou de pólen e néctar. De acordo com Coll e Guershon (2002), o comportamento de predadores zoofitófagos que são capazes de trocar facultativamente a alimentação entre presa e planta, é extremamente importante no controle biológico, pois permite a manutenção desses inimigos naturais durante os períodos de escassez de presas. A espécie $P$. nigrispinus pode sobreviver até 15 dias sem a presença de presas, alimentando-se de folhas de eucalipto (HOLTZ et al., 2009). Segundo os autores esse fato demonstra que a alimentação com plantas é uma estratégia bem sucedida para a sobrevivência desse predador em escassez de alimentos.

Além disso, a ocorrência dos inimigos naturais em povoamentos florestais homogêneos pode estar associada também à presença de fragmentos florestais nativos próximos as plantações de eucalipto, os quais podem atuar como reservatório desses predadores.

A presença dos predadores relatados no presente trabalho é uma alternativa no Manejo Integrado de Pragas e revela o potencial de uso desses inimigos naturais em surtos de $N$. nyseus. A espécie $B$. tabidus é

FIGURA 1: Exemplares adultos de predadores (A) Podisus nigrispinus (Hemiptera: Pentatomidae). (B) Brontocoris tabidus (Hemiptera: Pentatomidae). (C) Alcaeorrhynchus grandis (Hemiptera: Pentatomidae), coletados em plantações de Eucalyptus saligna, na região de São Sepé, RS. Maio de 2011.

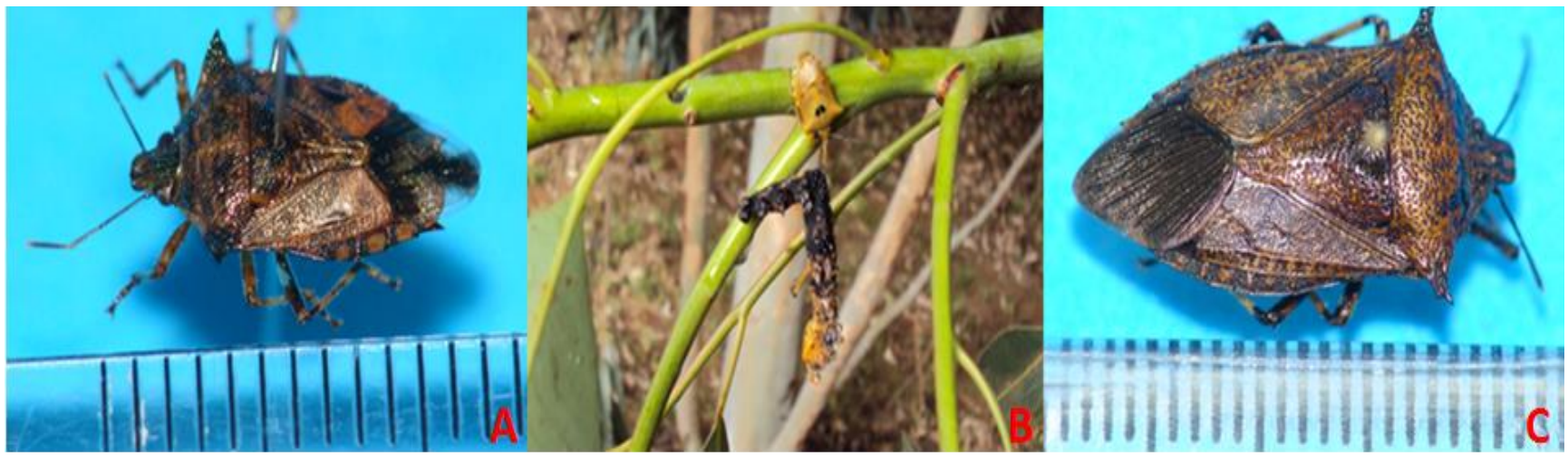


relatada pela primeira vez como predadora de lagartas de $N$. nyseus. As espécies $P$. nigrispinus e $A$. grandis são registradas em lagartas de $N$. nyseus para o Estado do Rio Grande do Sul.

\section{Agradecimentos}

À Dra. Jocelia Grazia da UFRGS pela identificação dos predadores. Ao Dr. Sinval Silveira Neto do Museu de Entomologia da Universidade de São Paulo-USP pela identificação da espécie $N$. nyseus.

\section{Referências}

ALMEIDA, G. A.; FADINI, M. A. M.; ZANUNCIO, J. C.; ANDRADE, G. S. Phytophagy on eucalyptus plants increases the development and reproduction of the predator Podisus nigrispinus (Hemiptera: Pentatomidae). Acta Scientiarum Agronomy, Maringá, v. 33, p. 231-235, 2011.

BARCELLOS, A.; GRAZIA, J. Revision of the genus Poriptus St 1 (Hemiptera: Heteroptera: Pentatomidae: Pentatominae). Zootaxa, Auckland, v. 1821, p. 25-36, 2008.

BUNDE, P. R. S. Levantamento da diversidade de percevejosdo-mato (Heteroptera: Pentatomoidae) na Serra do Sudeste do Rio Grande do Sul, Brasil. 2005. 72 f. Dissertação (Mestrado em Biologia Animal) - Universidade Federal do Rio Grande do Sul, Porto Alegre. 2005.

COLL, M.; GUERSHON, M. Omnivory in terrestrial arthropods: mixing plant and prey diets. Annual Review of Entomology, Palo Alto, v. 47, p. 267-297, 2002.

COSTA, E. C.; BOGORNI, P. C.; BELlOMO, V. H. Percevejos coletados em copas de diferentes espécies florestais. Ciência Florestal, Santa Maria, v. 5, n.1, p.123-128, 1995.

DEBACH, P.; ROSEN, D. Biological control by natural enemies. New York: Cambridge University Press, 1991. 440 p.

DE CLERCQ, P. Predaceous stinkbugs (Pentatomidae: Asopinae). In: SCHAEFER, C. W.; PANIZZI, A. R. (Ed.). Heteroptera of economic importance. Cambridge: Cambridge University, 2000. p. 737-789.

GARleT, J.; ROMAN, M.; COSTA, E. C. Pentatomídeos (Hemiptera) associados a espécies nativas em Itaara, RS, Brasil. Biotemas, Florianópolis, v. 23, n. 1, p. 91-96, 2010.

GRAZIA, J.; SILVA, E. J. E.; GARCIA, E.; SCHWERTNER, C. F.; BARCELLOS, A. Plantas utilizadas por percevejos-do-mato (Hemiptera:Pentatomoidea) no litoral norte do Rio Grande do Sul. In: CONGRESSO BRASILEIRO DE ZOOLOGIA, 24, 2004, Resumos... Brasília: SBZ, 2004. p. 131-131.

GUTIERREZ, C. T. Desenvolvimento e reprodução do predador Brontocoris tabidus (Heteroptera: Pentatomidae) no campo após exposição à deltametrina. 2006. 88 f. Dissertação (Mestrado em Entomologia) - Universidade Federal de Viçosa, Viçosa. 2006.

HOLTZ,A. M.; ALMEIDA, G. D.; FADINI, M.A. M.; ZANUNCIOJUNIOR, J. S.; ZANUNCIO, T. V.; ZANUNCIO, J. C. Survival and reproduction of Podisus nigrispinus (Heteroptera:Pentatomidae): effects of prey scarcity and feeding. Chilean Journal of Agricultural Research, Chillán, v. 69, p. 468-472, 2009.

JUSSELINO FILHO, P.; ZANUNCIO, J. C.; GUEDES, R. N. C.; FRAGOSO, D. B. Desarrollo y reproducción del predador Brontocoris tabidus (Heteroptera: Pentatomidae) alimentado com larvas do Tenebrio molitor (Coleoptera: Tenebrionidae). Revista Colombiana de Entomologia, Bogotá, v. 27, p. 45-48, 2001.

LEMOS, W. P. Efeito de diferentes presas no desenvolvimento das estruturas reprodutivas e na reprodução do predador Podisus nigrispinus (Heteroptera: Pentatomidae). 2001. $106 \mathrm{f}$. Dissertação (Mestrado em Entomologia) - Universidade Federal de Viçosa, Viçosa. 2001.

LINK, D.; GRAZIA, J. Pentatomídeos da região central do Rio Grande do Sul (Heteroptera). Anais da Sociedade Entomológica do Brasil, Londrina, v. 16, n. 1, p. 115-129, 1987.

MATESCO, V. C.; BIANCHI, F. M.; CAMPOS, L. A.; GRAZIA, J. Egg ultrastructure of two species of Galgupha Amyot \& Serville, with a discussion of the eggs and oviposition patterns of thyreocorid and allied groups (Hemiptera: Heteroptera: Pentatomoidea: Thyreocoridae). Zootaxa, Auckland, v. 3247, p.43-51, 2012.

MATESCO, V. C.; FORTES, N. D. F.; GRAZIA, J. Imaturos de pentatomídeos (Hemiptera, Heteroptera): morfologia e biologia de Acrosternum obstinatum. Iheringia, Série Zoologia, Porto Alegre, v. 93, p. 81-88, 2003.

MOREnO, J. A. Clima do Rio Grande do Sul. Porto Alegre: Secretaria da Agricultura, 1961. 73 p.

RUBERSON, J. R.; TAUBER, M. J.; TAUBER, C. A. Plant feeding by Podisus maculiventris (Heteroptera: Pentatomidae): Effect on survival, development and preoviposition period. Environmental Entomology, College Park, v. 15, p. 894-897, 1986.

SCHMIDT, L. S.; BARCELLOS, A. Abundância e riqueza de Heteroptera (Hemiptera) do Parque Estadual do Turvo, sul do Brasil: Pentatomoidea. Iheringia, Série Zoologia. Porto Alegre, v. 97, n. 1, p. 73-79, 2007.

TORRES, J. B.; BOYD, D. W. Zoophytophagy in predatory Hemiptera. Brazilian Archives of Biology and Technology, Curitiba, v. 52, p. 1199-1208, 2009.

TORRES, J. B.; ZANUNCIO, J. C.; MOURA, M. A. The predatory stinkbug Podisus nigrispinus: biology, ecology and augmentative releases for lepidoperan larval control in Eucalyptus forests in Brazil. Perspective in Agriculture Veterinary Science Nutrition and Natural Resources, Tokyo, v. 15, p. 1-18, 2006.

WEILER, L.; FERRARI, A.; GRAZIA, J. Contributions to the knowledge of Euschistus (Lycipta) with the description of E. (L.) riograndensis sp. nov. (Hemiptera: Heteroptera: Pentatomidae: Pentatominae: Carpocorini). Zootaxa, Auckland, v. 3067, p. 59-64, 2011.

ZANUNCIO, J. C.; ALVES, B. A.; ZANUNCIO, T. V. Hemipterous predators of Eucalypt desfoliator caterpillars. Forest Ecology and Management, Maryland Heights, v. 65, p. 65-73, 1994.

ZANUNCIO, J. C.; GUEDES, R. N. C.; OLIVEIRA, H. N.; ZANUNCIO, T. V. Uma década de estudos com percevejos predadores: conquistas e desafios. In: PARRA, J. R.; BOTELHO, P. S. M.; CORREAA-FERREIRA, B. S.; BENTO, J. S. (Ed.). Controle biológico no Brasil: parasitoides e predadores. São Paulo: Manole, 2002. p. $495-505$. 